\title{
ESTUDO DA DINÂMICA DE FLUIDIZAÇÃO DE UM SISTEMA COMPOSTO AREIA- GÁS-BIOMASSA
}

\author{
M. C. S. SANT'ANNA ${ }^{1 *}$, M. L. A. TAVARES ${ }^{1}$, S. M. SARMENTO' ${ }^{1}$, G. F. SILVA ${ }^{2}$, R. A. \\ MEDRONHO $^{3}$, S. LUCENA ${ }^{1}$ \\ ${ }^{1}$ Universidade Federal de Pernambuco, Departamento de Engenharia Química \\ ${ }^{2}$ Universidade Federal de Sergipe, Departamento de Engenharia Química \\ ${ }^{3}$ Universidade Federal do Rio de Janeiro, Escola de Química, Departamento de Engenharia Química \\ *e-mail: mikelecandida@gmail.com
}

\begin{abstract}
RESUMO
Leitos fluidizados podem ser utilizados em inúmeros processos industriais, dentro dos quais, destacam-se a combustão e/ou gaseificação de biomassa, estes equipamentos promovem um maior contato sólido-gás. Objetivando entender a dinâmica das partículas durante a fluidização, foram realizadas simulações para dois sistemas contendo gás-biomassa-areia, onde variou-se o diâmetro das partículas da fase sólida, verificando a influência desta alteração na fluidização das partículas. Para realizar as simulações foi utilizado o software ANSYS FLUENT 15.0, foi adotada a abordagem Euleriana, com a teoria cinética granular. A velocidade de entrada do gás foi mantida a $0,38 \mathrm{~ms}^{-1}$. Entre os sistemas simulados, o sistema A apresentou fluidização difícil quando comparado com o sistema $\mathrm{B}$, onde a fluidização ocorreu mais facilmente e os efeitos da segregação foram atenuados. Compreender a hidrodinâmica nestes sistemas é importante para otimizar os processos.
\end{abstract}

\section{INTRODUÇÃO}

A hidrodinâmica de reatores de leito fluidizados tem atraído diversos pesquisadores por décadas para entender as complexas interações entre gás e partículas Berruti et al., 1995; Samuelsberg e Hjertager, 1995; Benyahia, 2000; Goldschmidt, 2001; Almuttahar e Taghipour, 2008; Armstrong et al., 2010; Tagliaferri et al. (2013).

Segundo Hodapp (2009) a terminologia fluidização provém do fato de um sólido, quando finamente dividido, se comportar como um fluido quando arrastado por um gás ou um líquido. Porém este é um fenômeno bastante complexo, podendo ser caracterizado pelo grau de influência do fluido sobre a fase sólida de um leito.
Neste tipo de equipamento utiliza-se um elemento como a areia para servir de leito, o qual será aquecido e mantido em suspensão (fluidizado) por um fluido, que pode ser o ar.

$\mathrm{Na}$ fluidização a força da gravidade agindo nas partículas é compensada pelas forças de arraste exercidas pelo escoamento local do fluido. O escoamento local do fluido é diferente para cada partícula, fazendo o comportamento de cada partícula único (GIBILARO, 2001; MARINI, 2008).

Estes equipamentos podem ser utilizados para a conversão de biomassa em energia.

Avaliar o comportamento da fluidização levando em consideração a presença do material a ser gaseificado foi um diferencial abordado neste trabalho, na literatura os autores Taghipuor et al. (2005), 
Herzog et al. (2012), Tagliafferi et al. (2013) realizam uma simplificação e avaliam o sistema de fluidização utilizando técnicas de CFD somente levando em consideração a presença da areia, já que esta é a mais densa, apresenta-se em maior quantidade no leito e é a responsável pela homogeneização da queima durante o processo de gaseificação. A simplificação objetiva reduzir a complexidade do experimento em CFD.

\section{METODOLOGIA}

O software comercial utilizado foi $\mathrm{O}$ ANSYS FLUENT 15.0 e ANSYS CFX 15.0.

A geometria é uma simplificação $2 \mathrm{D}$ do gaseificador de leito fluidizado borbulhante disponível no Laboratório de Controle Avançado e Otimização de Processos (LACO-UFPE) (Figura 1). A largura do leito e altura do leito foram de 0,58 e $2,00 \mathrm{~m}$, respectivamente. A malha utilizada nas simulações possui 72.500 elementos e foi obtida através de um teste de independência de malha descrito em Sant'Anna (2015).

Figura 1 - Geometria Construída em 2D

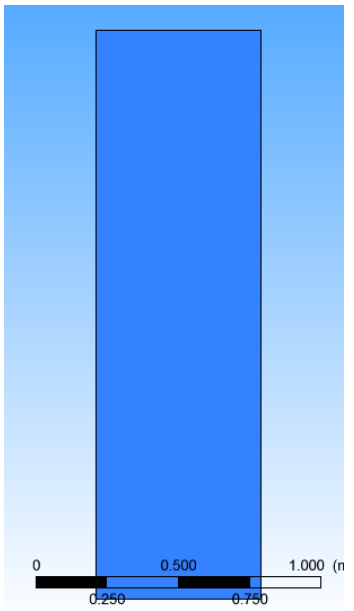

Fonte: Autor (2015).

Para avaliar a dinâmica de fluidização, a fase sólida foi composta por material inerte (areia) e o combustível (biomassa) e pelo gás na fase fluida. As propriedades da biomassa, da areia e do gás foram apresentadas na Tabela 1. A velocidade de entrada do gás utilizada nestas simulações foi fixada em $0,38 \mathrm{~ms}^{-1}$. Os dados para areia e gás estavam disponíveis no ANSYS FLUENT (2011) e para a Biomassa foi utilizado os citados por Oliveira (2012).

Tabela 1- Propriedades das fases sólida e gasosa a $300 \mathrm{~K}$ e 1 atm.

\begin{tabular}{|c|c|c|}
\hline Material & $\begin{array}{l}\text { Propriedade } \\
\end{array}$ & Valor \\
\hline Areia & $\begin{array}{l}\text { Densidade }\left(\mathrm{kg} \mathrm{m}^{-3}\right) \\
\text { Diâmetro da partícula } \\
(\mu \mathrm{m})\end{array}$ & $\begin{array}{c}2,50 \times 10^{3} \\
250 / 275\end{array}$ \\
\hline Biomassa & $\begin{array}{l}\text { Densidade }\left(\mathrm{kgm}^{-3}\right) \\
\text { Diâmetro da partícula } \\
(\mu \mathrm{m})\end{array}$ & $\begin{array}{c}1,43 \times 10^{3} \\
350 / 500\end{array}$ \\
\hline Air & $\begin{array}{l}\text { Densidade }\left(\mathrm{kg} \mathrm{m}^{-3}\right) \\
\text { Calor especifico } \\
\left(\mathrm{JKg}^{-1} \mathrm{~K}^{-1}\right) \\
\text { Condutividade Térmica } \\
\left(\mathrm{Wm}^{-1} \mathrm{~K}^{-1}\right) \\
\text { Viscosidade }\left(\mathrm{kgm}^{-1} \mathrm{~s}^{-1}\right)\end{array}$ & $\begin{array}{l}0,13 \times 10^{1} \\
1,01 \times 10^{3} \\
2,40 \times 10^{-2} \\
7,89 \times 10^{-5}\end{array}$ \\
\hline
\end{tabular}

Nos dois sistemas simulados foram mantidas as frações de sólido $(\phi)$ e vazios $(\varepsilon)$ respectivamente iguais a $60 \%$ e $40 \%$. Nestes estudos, fixou-se a composição da fase sólida, porém variou-se o diâmetro das partículas (Tabela 2) visando-se verificar o efeito deste parâmetro sobre à dinâmica do leito fluidizado.

Tabela 2- Composição da fase sólida para o Sistema ar-areia-biomassa

\begin{tabular}{llccc}
\hline & \multicolumn{4}{c}{ Propriedade } \\
\cline { 2 - 5 } & Material & $\phi(\%)$ & $\begin{array}{c}\text { Densidade } \\
\left(\mathrm{kg} \mathrm{m}^{-3}\right)\end{array}$ & $\begin{array}{c}\text { Diametro } \\
(\mu \mathrm{m})\end{array}$ \\
\multirow{2}{*}{ A } & Areia & 51,00 & $2,50 \times 10^{3}$ & $2,75 \times 10^{2}$ \\
& Biomassa & 0,90 & $1,41 \times 10^{3}$ & $5,00 \times 10^{2}$ \\
& Areia & 51,00 & $2,50 \times 10^{3}$ & $2,50 \times 10^{2}$ \\
& Biomassa & 0,90 & $1,41 \times 10^{3}$ & $3,50 \times 10^{2}$ \\
\hline
\end{tabular}

O modelo de turbulência utilizado foi o k- $\varepsilon$, escolhido através da realização de um 
teste de modelo, onde se avaliou o comportamento dos modelos k- $\varepsilon$ e SST, descrito em Sant'Anna (2015).

A simulação do leito fluidizado foi realizada resolvendo as equações da conservação de massa, momentum e energia através da utilização do software de CFD.

A abordagem Euleriana foi adotada neste trabalho, juntamente com a Teoria Cinética Granular descrita em ANSYS FLUENT (2011).

Equação da conservação de massa (o subscrito i pode ser para fase gás ou sólida):

$$
\frac{\partial}{\partial t}\left(\alpha_{i} \rho_{i}\right)+\nabla\left(\alpha_{i} \rho_{i} \vec{v}_{i}\right)=0
$$

Equação da conservação de momentum para fase gás (g) e fase sólida (s):

$$
\begin{aligned}
& \frac{\partial}{\partial t}\left(\alpha_{g} \rho_{g} \vec{v}_{g}\right)+\nabla\left(\alpha_{g} \rho_{g} \vec{v}_{g}^{2}\right)= \\
& -\alpha_{g} \nabla p+\nabla \bar{\tau}_{g}+\alpha_{g} \rho_{g} \vec{g}+K_{g s}\left(\vec{v}_{g}-\vec{v}_{s}\right) \\
& \frac{\partial}{\partial t}\left(\alpha_{s} \rho_{s} \vec{v}_{s}\right)+\nabla\left(\alpha_{s} \rho_{s} \vec{v}_{s}^{2}\right)= \\
& -\alpha_{s} \nabla p-\nabla p_{s}+\nabla \overline{\bar{\tau}}_{s}+\alpha_{s} \rho_{s} \vec{g}+K_{g s}\left(\vec{v}_{g}-\vec{v}_{s}\right)
\end{aligned}
$$

As equações constitutivas foram necessárias para o fechamento das equações governantes. A viscosidade da partículas foi calculada pela Teoria Cinética Granular, as correlações foram descritas na Tabela 3.

Tabela 3- Equações de viscosidade

\begin{tabular}{cc}
\hline Viscosidade & Modelo \\
\hline Viscosidade cisalhante & Syamlal-Obrien, 1989 \\
Viscosidade Colisional & Gidaspow et al., 1994 \\
Viscosidade cinética & Syamlal et al., 1993 \\
Viscosidade cisalhante & Schaeffer, 1987 \\
friccional & Lun et al., 1984 \\
Viscosidade global &
\end{tabular}

Segundo Ansys (2011) é preferível a utilização do modelo Syamlal-O'Brien (1989) para a força de arraste quando a viscosidade cinética e cisalhante são modeladas pelas equações de Syamlal et al. (1993). Este modelo é baseado em medidas de velocidade terminal das partículas em leitos fluidizados com correlações em função da fração volumétrica e do número de Reynolds relativo.

As equações utilizadas pelo modelo de Syamlal-O'Brien (1989) encontram-se descritas em ANSYS FLUENT (2011).

O valor do coeficiente de restituição partícula-partícula foi igual a 0,9 como utilizado por Taghipuor et al. (2005), Adamczyk et al. (2013), Cornelissen et al. (2007), Pain et al. (1984) e Faísca (2013).

A areia utilizada no leito do gaseificador é do tipo quartzosa, este material é necessário para melhorar a estabilidade térmica e dinâmica do sistema.

A resolução do problema foi realizada de forma segregada utilizando o método Phase Coupled SIMPLE para solução do acoplamento pressão-velocidade. Foram realizados testes para alcançar um valor de time-step de modo a obter o menor número de Courant. Convencionou-se o passo de tempo (time-step) fixo de $5.10^{-5}$ segundos com 100.000 iterações (5,00 s de simulação).

\section{RESULTADOS}

A Figura 2 apresenta os perfis longitudinais de fração volumétrica do ar, areia e biomassa para os diferentes tempos de simulação $(0,02 ; 1,25 ; 2,50 ; 3,75$ e $5,00 \mathrm{~s})$, para o sistema A (apresentado na Tabela 2). A velocidade utilizada foi de $0,38 \mathrm{~ms}^{-1}$. 
Figura 2- Perfis longitudinais de fração volumétrica (Sistema $\mathrm{A}, \mathrm{v}_{\mathrm{g}}=0,38 \mathrm{~ms}^{-1}$ ).

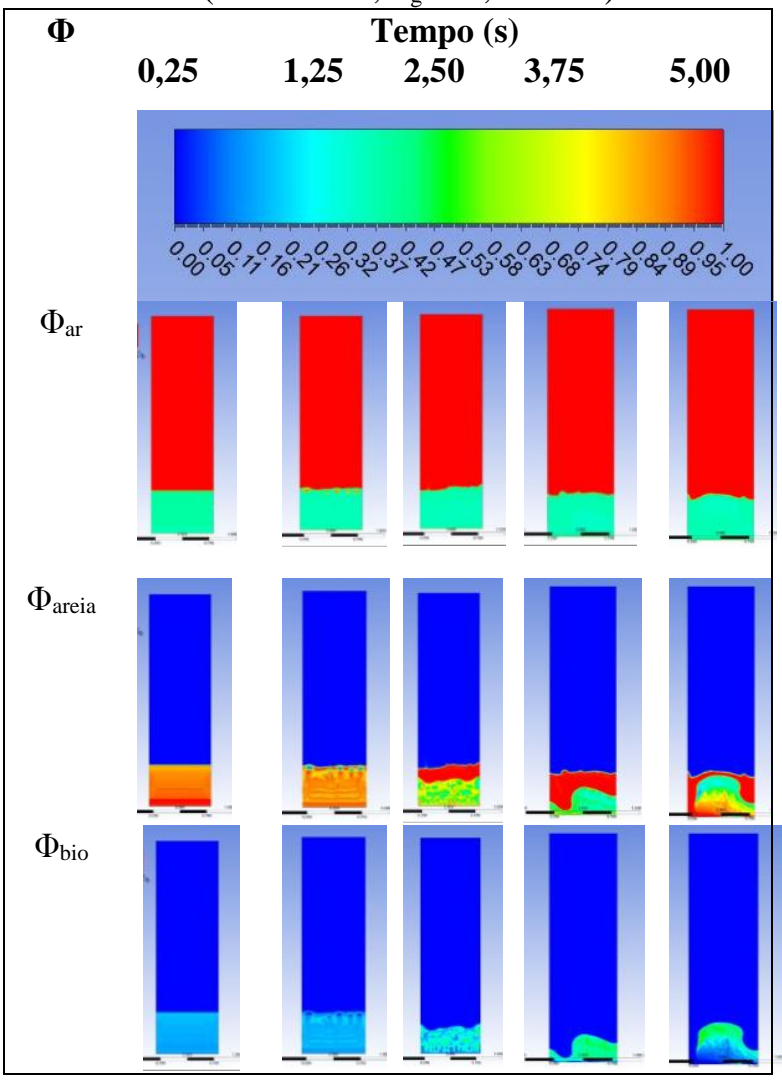

Fonte: Autor (2015).

É possível observar que com o passar do tempo ocorre à segregação entre a areia e a biomassa. Segundo Oliveira (2012) para misturas em que as diferenças entre os diâmetros da biomassa e areia são maiores, ocorre forte segregação destas misturas, a queda de pressão não atinge um patamar ao longo da fluidização. A fluidização é considerada "pobre", com formação de canais preferenciais, formação de slugging (grandes bolhas) e forte segregação. Este fato foi verificado experimentalmente pelo autor através das fortes oscilações na queda de pressão do leito na região de fluidização completa.

A Figura 3 apresenta os perfis longitudinais de fração volumétrica do ar, areia e biomassa para os diferentes tempos de simulação $(0,02 ; 1,25 ; 2,50 ; 3,75$ e 5,00 s $)$.
Figura 3- Perfis longitudinais de fração volumétrica (Sistema $\mathrm{B}, \mathrm{v}_{\mathrm{g}}=0,38 \mathrm{~ms}^{-1}$ ).

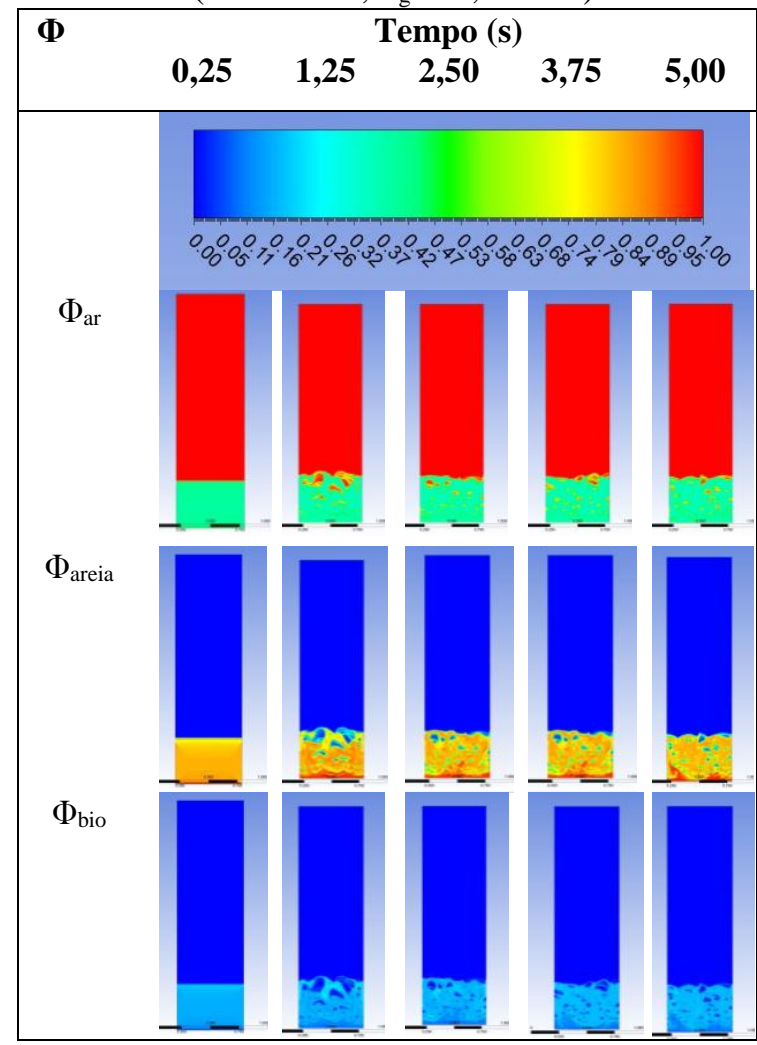

Fonte: Autor (2015).

É possível verificar neste sistema que a fluidização ocorre mais facilmente que no sistema A. Segundo Oliveira (2012) misturas que possuem diâmetros de biomassa e areia próximos tem por consequência o efeito da segregação atenuado.

A Figura 4 apresenta os perfis longitudinais pressão e os valores para a queda de pressão para os diferentes tempos de simulação $(0,02 ; 2,50$ e $5,00 \mathrm{~s})$, para o sistema A e B (apresentado na Tabela 3.8). A velocidade utilizada foi de $0,38 \mathrm{~ms}^{-1}$. 
Figura 4- Perfis de longitudinais de pressão (Sistemas A e B, $\mathrm{v}_{\mathrm{g}}=0,38 \mathrm{~ms}^{-1}$ ).

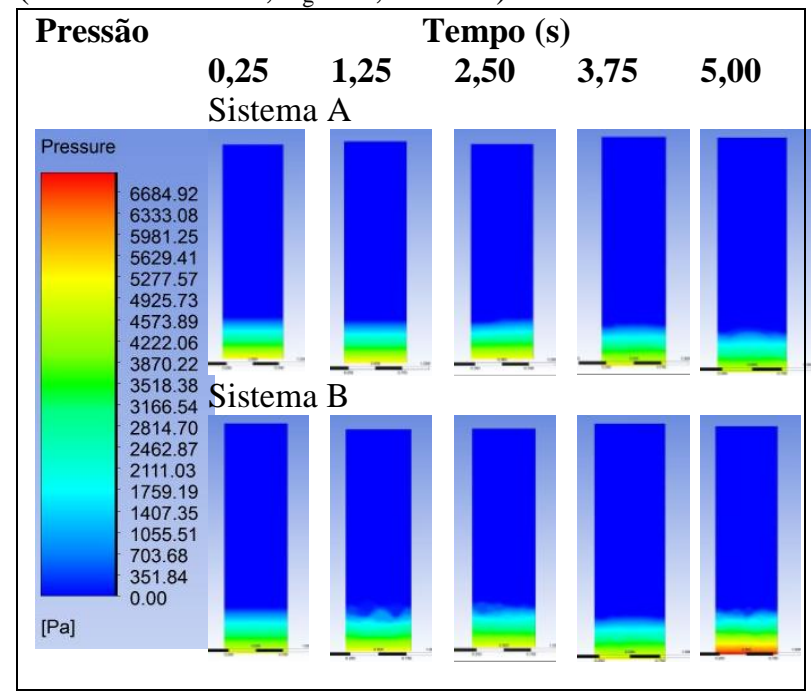

Fonte: Autor (2015).

Segundo Oliveira (2012) uma das condições necessárias para a operação do leito fluidizado é a obtenção de uma mistura que mantenha uma boa homogeneidade entre a biomassa e a areia. A utilização da técnica de fluidodinâmica é importante para se analisar o efeito da mistura de biomassa e areia e para que o efeito da segregação seja minimizado.

O conhecimento da velocidade de mínima fluidização está ligada a eficiência do leito fluidizado, pois uma vez abaixo da velocidade mínima o leito não fluidiza e muito acima da velocidade mínima ocorre o arraste das partículas presentes no leito.

Ainda segundo Oliveira (2012) nenhuma das equações convencionais de $\mathrm{U}_{\mathrm{mf}}$ foram capazes de fornecer previsões confiáveis, especialmente para misturas de biomassa e areia. Clarke et al. (2005) relataram que as correlações existentes na literatura não foram capazes de prever a $U_{\mathrm{mf}}$ de misturas binárias de esferas de vidro e serragem. Essas correlações podem depender fortemente das partículas utilizadas especificamente no desenvolvimento das expressões.

\section{REFERÊNCIAS}

ALMUTTAHAR, A.; TAGHIPOUR, F. Computational fluid dynamics of high density circulating fluidized bed riser: study of modelling parameters, Powder Technol, v.185, p.11-23, 2008.

ANDRADE, R. B. Gaseificação de Biomassa: Uma Análise Teórica e Experimental. Tese de Doutorado. Universidade Federal de Itajubá. Itajubá. 2007.

ANSYS FLUENT 13.0 - Theory Guide. Ansys Inc. USA, 2011.

ARMSTRONG, L.M.; GU, S.; LUO, K.H. Study of wall-to-bed heat transfer in a bubbling fluidised bed using the kinetic theory of granular flow. International Journal of Heat and Mass Transfer, v.53, p.4949-4959, 2010.

BENYAHIA, S.; ARASTOOPOUR, H.; KNOWLTON, T.; MASSAH, H. Simulation of particles and gas flow behaviour in the riser section of a circulating fluidized bed using the kinetic theory approach for the particulate phase, Powder Technol, v.112, p. 24-33, 2000.

BERRUTI，F.; CHAOUKI，J.; GODFROY, L.; PUGSLEY, T.; PATIENCE, G. Hydrodynamics of circulating fluidized bed risers: a review, Can. J. Chem. Eng, v.73 (5), p.579- 602, 1995.

GIBILARO, L. G. Fluidization Dynamics. Oxford: Butterworth-Heinemann, 2001.

GOLDSCHMIDT, M.; KUIPERS, J.; VAN SWAAIJ, W. Hydrodynamic modelling of dense gas-fluidized beds using the kinetic theory of granular flow: effect of restitution coefficient on bed dynamics, Chem. Eng. Sci, v.56, p.571, 2001.

HERZOG, N.; SCHREIBER, M.; EGBERS, C.; KRAUTZ, H. J. A comparative study of different CFD-codes for numerical simulation of gas-solid fluidized bed hydrodynamics. Computers and Chemical Engineerin, v.39, p.41-46, 2012. 
HODAPP, M. J. Modelagem e simulação de um leito fluidizado: um estudo comparativo. Dissertação de Mestrado. Faculdade de Engenharia Química. Universidade Estadual de Campinas. Campinas - SP. 2009.

MARINI, F. Simulação de um leito fluidizado aplicando a técnica CFD baseada na teoria cinética do escoamento granular. Dissertação de Mestrado. Faculdade de Engenharia Química. UNIVERSIDADE ESTADUAL DE CAMPINAS. Campinas - SP. 2008.

SAMUELSBERG, A.; HJERTAGER, B. An experimental and numerical study of flow patterns in a circulating fluidized bed reactor, Int. J. Multiphase Flow, v.22, p.575-591, 1995.

TAGLIAFERRI, A.; MAZZEI, L.; LETTIERI, P.; MARZOCCHELLA, A.; OLIVIERI, G.; SALATINO, P. CFD simulation bubbling fluidized bidispese mixtures: Effect of integration method sand restitution coefficient. Chemical Engineering Science, n.102, p.324-334, 2013.

TAGHIPOUR, F.; ELLIS, N.; WONG, C. Experimental and computational study of gas-solid fluidized bed hydrodynamics. Chemical Engineering Science, v.60, p.6857-6867, 2005.

OLIVEIRA, T. J. P. Aspectos fluidodinâmicos de misturas binárias de resíduo de tabaco e areia em Leito Fluidizado. Universidade Federal de Uberlândia. Uberlândia-MG, 2012. 